\title{
Social media embraces suicide prevention
}

$\mathrm{D}$ aniel Reidenberg was scanning Facebook for his suicide-prevention organization when he saw the post from a clearly suicidal young woman. A new widow with a young baby, she had taken to social media to announce her intention to kill herself and her child.

Responding to suicidal messages and threats is a growing challenge for social media organizations. It's also one in which Reidenberg, the executive director of the United States-based prevention organization Suicide Awareness Voices of Education (SAVE), is deeply involved.

It was a Friday evening in the spring of 2014 when Reidenberg noticed the post. Because he had been working with Facebook, Google, Tumblr, Twitter and other digital technology companies on suicide prevention, Reidenberg knew exactly what to do: he contacted Facebook's safety centre. There was no indication in the post where the young woman was, but he knew Facebook might be able to find out.

Within "two to five minutes," Reidenberg told the International Association for Suicide Prevention's annual conference in Montréal, Quebec, Facebook employees had determined the woman had posted her message from an Internet café in a small village in South America. They contacted local police. Holding a picture from the woman's Facebook page, police officers walked through the village.

Ten minutes after the woman left the café with her baby, police found her. "Because they had a picture, they were able to find her and get her into a hospital and get her child care," Reidenberg said.

Reidenberg told the startling anecdote in response to questions from CMAJ about his June 17 presentation on the power of technology and social media to prevent suicide. Using digital technology to prevent suicide was the conference's key theme.

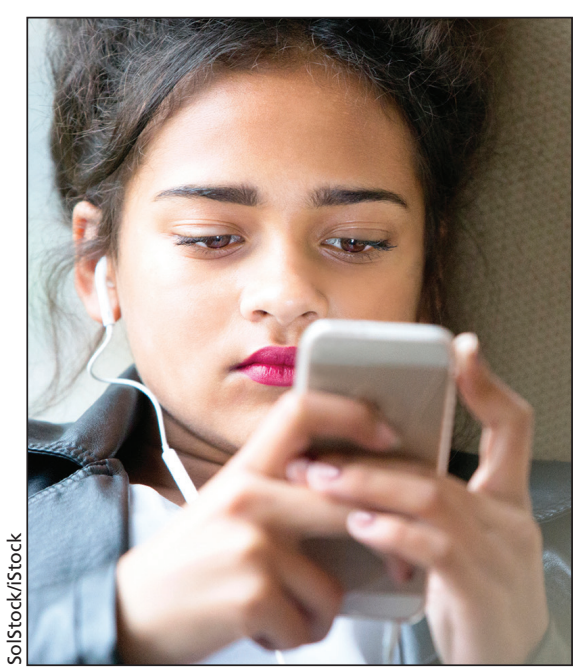

In the United States, a Facebook post indicating suicidal thoughts or intentions prompts an automatic response and followup from Facebook.

With 2 billion people using social media and 500 million people posting on Facebook alone, there is "a lot of opportunity for intervention and prevention," says Reidenberg. "It's critical that everyone start looking at this new era with technology being involved in the field of suicide prevention. It isn't just going to a doctor's office any longer it is that "plus'."

Facebook, Google and their competitors are increasingly collaborating in suicide prevention. Although none of these companies wants to be in the prevention business, Reidenberg says, people use their platforms and devices to communicate cries for help and to research ways of killing themselves.

In a few short years since some of the big social media companies began meeting periodically at Facebook's California head office in 2011 to brainstorm about what to do, the world's largest social network has rolled out a direct intervention. In 25 of the 50 American states, when a user posts a message on Facebook containing a phrase that its algorithms flag as indi- cating suicidal thoughts or intentions, a banner pops up on the user's page. Friends viewing the post can also click on an icon that reports the message, sending it to the Facebook safety centre to review.

The automatic banner asks whether the user needs help and provides the number of the National Suicide Prevention Lifeline. Facebook then contacts the user and offers to connect him or her to the distress line. The message includes tips and links to support videos aligned with best prevention practices. Facebook may also freeze the user's page until the person in distress has interacted with the support applications.

This prevention program will expand to the remaining states by the end of the year, Reidenberg says. The company is piloting it in two other undisclosed countries, and it will eventually be available in Canada and globally.

One of the challenges in bringing this approach to Canada is the lack of a national suicide prevention strategy and infrastructure here, says conference organizer Brian Mishara, the director of the Centre for Research and Intervention on Suicide and Euthanasia (CRISE) at the Université du Québec à Montréal. Particularly lacking is a single national number that anyone who is suicidal can call, he says.

Facebook's new program raises "thorny" ethical issues about its users' right to communicate thoughts they may or may not intend to act upon, one psychiatrist attending the conference suggested.

Companies are wrestling with the privacy issue, Reidenberg acknowledged. But he reminded the audience that once someone posts anything on a social media site - even if it's personal health information - it is no longer private. Laura Eggertson, Montréal, Que.

CMAJ 2015. DOI:10.1503/cmaj.109-5104 